\title{
ScreenTape as a tool for the rapid differentiation of Mycobacterium tuberculosis isolates
}

With over 9 million new cases annually and an estimated prevalence of 14.4 million, tuberculosis $(\mathrm{TB})$ remains a public health problem of global proportions (WHO, 2008). The recent emergence of drug-resistant strains of Mycobacterium tuberculosis that cannot be cured by the currently available anti-TB drugs has intensified the need to improve disease control (Dorman \& Chaisson, 2007). Prompt detection and treatment of infectious cases is required to prevent onward transmission. Genotyping $M$. tuberculosis strains can aid TB control through early identification of transmission events, permitting targeted screening and intensified case finding to contain the outbreak. This is particularly important when dealing with drugresistant disease where patients on standard anti-TB drug therapy may remain infectious and a source of onward transmission. To be of best use to public health practitioners, genotyping data must be available within days of a case being notified. Genotyping techniques based on PCR amplification of specific regions of the genome offer a rapid way to differentiate bacterial strains. One of the most discriminatory of these techniques for differentiating M. tuberculosis isolates is mycobacterial interspersed repetitive unit-variable-number tandem repeats (MIRU-VNTR) (Mazars et al., 2001; Supply et al., 2001; Kremer et al., 2005). In this method, loci, at which a variable number of repeats may be present, are amplified and the number of repeats is determined from the length of the resulting amplicon. A 12-locus MIRUVNTR method (Supply et al., 2000; Mazars et al., 2001) has been used in TB public health surveillance systems, for example in the United States (National TB Controllers Association, 2004) and in the UK (Health Protection Agency Centre for Infections, 2008). A standardized method for MIRUVNTR typing of $M$. tuberculosis has recently been established and a panel of 15 loci is now recommended for routine genotyping of $M$. tuberculosis isolates (Supply et al., 2006). The repeats from these 15 loci range in size from 51 to $111 \mathrm{bp}$ and the amplicons obtained following PCR of these loci may be between 77 and 1852 bp in length. According to the fragment size obtained from each locus, the number of repeats is determined and an allele is assigned - the final result is a 15-digit numerical code for each sample. Thus, accurate sizing is crucial for determining the number of repeats present at each locus. The traditional method of detecting and sizing MIRU PCR products involves manual gel electrophoresis and is a lengthy and laborious process. High-throughput analysis of MIRU-VNTR PCR products is possible using sophisticated apparatus such as an automated sequencer or HPLC (Supply et al., 2001; Evans et al., 2004). However, the capital outlay, maintenance costs and training requirements are beyond the budget of many public health laboratories, particularly in those countries where TB is endemic. A simple, rapid and cost-effective method that bridges the gap between manual gel electrophoresis and an automated sequencer would enable greater implementation of MIRU-VNTR to aid the control of TB.

In this study, we combined MIRU-VNTR typing with a new electrophoretic technology, ScreenTape (Lab901), and compared it to manual agarose gel electrophoresis to differentiate drug-resistant strains of M. tuberculosis. A commercial company specializing in $\mathrm{TB}$ genotyping, GenoScreen, was commissioned to undertake MIRU-VNTR analysis of the strains to provide an independent reference with which to assess the accuracy of the two methods. A panel of $20 \mathrm{M}$. tuberculosis clinical isolates was used for the study. The strains had been collected as part of a survey of TB drug resistance in Sudan. All isolates were subjected to drug susceptibility testing by the Löwenstein-Jensen (LJ) proportion method (Canetti et al., 1963) and all were resistant to the major anti-TB drug rifampicin. The isolates had been previously genotyped by spoligotyping using the Kamerbeek methodology (Kamerbeek et al., 1997). While this PCR-based technique is rapid, it has limited power to discriminate isolates of M. tuberculosis, and only two spoligotype patterns were found among the 20 drugresistant isolates. To determine whether resistance had emerged independently in these patients, or whether there is evidence to suggest ongoing transmission of drug-resistant TB in Sudan, it was necessary to genotype using the more discriminatory 15-loci MIRU-VNTR system (Supply et al., 2006).

DNA templates for genotyping were prepared by scraping colonies grown on LJ slopes into $400 \mu \mathrm{l}$ Tris/EDTA buffer and boiling for $20 \mathrm{~min}$. Fifteen PCRs, one for each locus, were set up for each isolate. The 300 PCR products were then run on manual gel electrophoresis and the ScreenTape system. The ScreenTape system comprises the ScreenTape, TapeStation and GeneTools software package. Each ScreenTape contains eight mini-gels for nucleic acid separation. One mini-gel is reserved for the appropriate molecular mass marker, allowing seven test samples to be run concurrently. Two sets of molecular mass markers were used in the study: ScreenTape D800 for fragments up to $800 \mathrm{bp}$, and ScreenTape D2K for larger fragments up to $2000 \mathrm{bp}$. One microlitre of each sample was mixed with loading buffer and placed into the TapeStation, where it was automatically loaded into the tape, electrophoresed and the resulting image was automatically analysed by GeneTools - a process that was completed in under $10 \mathrm{~min}$. For the manual analysis, the 20 PCR products from a given locus were run together in the same gel. Agarose gels were $20 \times 22.5 \mathrm{~cm}$ $(\mathrm{w} \times \mathrm{l})$ in size. Three lanes were used for 
the 100 bp molecular mass markers (Sigma). Two microlitres of each PCR product was electrophoresed for $5 \mathrm{~h}$ at $120 \mathrm{~V}$ on $3 \%$ molecular biology grade agarose (Electran; VWR) in 21 Trisborate-EDTA buffer at ambient temperature $\left(20-23{ }^{\circ} \mathrm{C}\right)$. The buffer was freshly made for each run. Bromophenol blue marker dye was used to monitor migration through the gel. Following staining with ethidium bromide, digital images of the gels were captured using the Multi Doc-It Digital Imaging System (UVP Inc.), and amplicon size was estimated using GeneTools v.3.07 software (Syngene). Manual analysis of the samples required over 1 week to complete; this included running two gels per day. The ScreenTape System proved easy to use and all 300 products were electrophoresed and sized in less than $10 \mathrm{~h}$.

Based on the DNA sizes obtained using gel electrophoresis and ScreenTape, the number of tandem repeats was determined by comparison to predicted fragment lengths for each locus and the corresponding allele number was assigned to each locus. Amplicon sizes and the assignment of allele number were compared. For the purpose of this study, the results received from GenoScreen were considered as the reference to which the in-house results were compared. These results were obtained by sending an aliquot of each DNA sample to GenoScreen, who performed the MIRU-VNTR PCR according to the standardized methodology. In this case, the PCR products were analysed using a highthroughput 96-capillary DNA Analyser (3730XL; Applied Biosystems).

Not all samples were amplified in the PCRs performed in-house. Where a locus did not amplify in a particular sample, it was excluded from the analysis for that sample. One locus (QUB-4156) was excluded completely as the amplicons produced were much smaller than predicted. Repeated PCR did not provide suitable bands and further investigation of the failure to amplify was not undertaken. The final number of results obtained by gel electrophoresis was 271. Comparison of amplicon sizes with those predicted by GenoScreen yielded a correlation coefficient of 0.9919 (Fig. 1) with $253(93.4 \%)$ of the alleles correctly assigned. When using

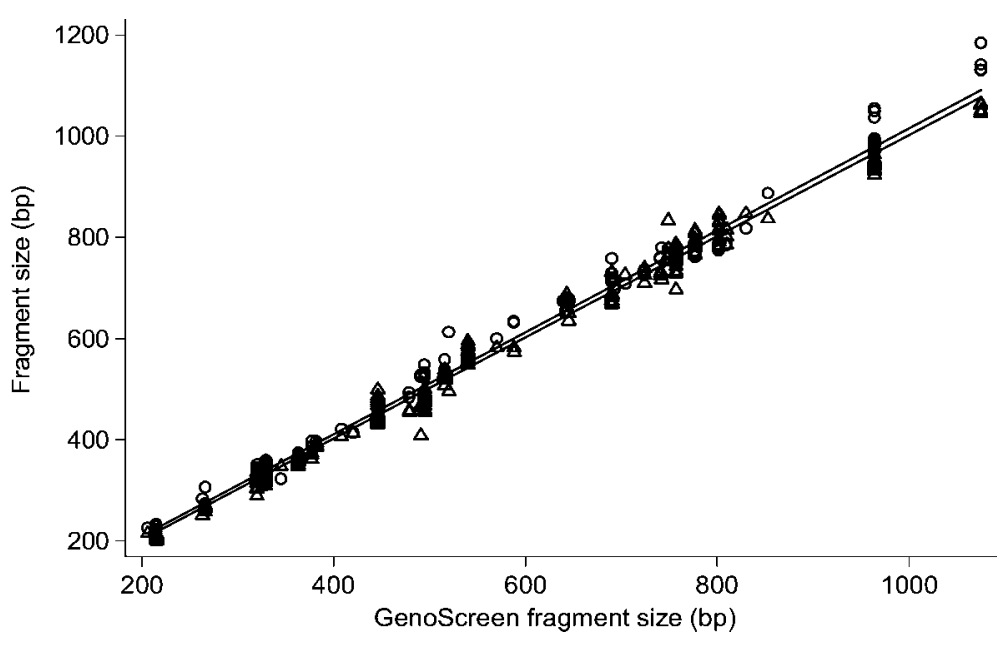

Fig. 1. Linear regression graph showing the relationship between fragment size based on GenoScreen results and the fragment size obtained by gel electrophoresis and ScreenTape. Sizes represented by hollow circles and triangles are for ScreenTape and gel electrophoresis, respectively.

ScreenTape, results were obtained for 272 of the samples, yielding a correlation coefficient of 0.9911 (Fig. 1) with 264 $(97.1 \%)$ of the alleles correctly assigned.

Typing of the 20 drug-resistant strains by MIRU-VNTR analysis provided 13 different genotypes. Seventeen isolates had previously been found by spoligotyping to match the Shared International Type (SIT) 25 of the Spoligotyping Database (SpolDB4; Institut Pasteur, Guadeloupe). By MIRU-VNTR typing, these isolates were found to comprise one cluster of five, and two clusters of two and eight individual MIRU types. Similarly, MIRUVNTR divided the three SIT 1 isolates into a cluster of two and a single strain. Thus, while spoligotyping suggested that a single genotype was responsible for concurrent outbreaks of multidrug-resistant (MDR)TB, MIRU-VNTR demonstrated that in fact more genotypes were involved. More detail regarding the origins of these strains and the epidemiology of drug-resistant TB in Sudan will be presented in a further article (in preparation).

The present study illustrates the value of the MIRU-VNTR technique in a country with a high burden of TB with an emerging problem of drug-resistant disease. To effect control of MDR-TB, it is necessary to know whether and where transmission of MDR-TB is occurring. If the MDR-TB strains have different genotypes then resistance is emerging concurrently in patients due to inadequate drug therapy and it is important that measures are taken to remedy the situation. The MIRU-VNTR typing for this study was performed in Europe as the capacity to undertake this work in Sudan does not exist. ScreenTape would appear to offer an alternative to the tedious, and somewhat unreliable, manual methods for assessing the genotypes of $M$. tuberculosis.

The ScreenTape system as used in this study provided rapid, simple implementation of MIRU-VNTR for genotyping M. tuberculosis. When compared to the traditional method of gel electrophoresis, it demonstrated improved speed and accuracy. The system proved easy to use and required little training. This contrasted with the preparation and running of gels for gel electrophoresis, where the sizing of the PCR fragments depended greatly upon the quality of the gel and/or of the gel image. Intra- and inter-gel variation was observed and some gels needed to be run more than once in order to achieve an interpretable result. The variation in the size of the PCR products obtained in this study, from 200 to over $1000 \mathrm{bp}$, made the measurement of small incremental repeats difficult when using visual recognition of bands. However, the performance of the manual electrophoresis could have been improved 
Table 1. Estimated 'hands-on' time in minutes of performing manual gel electrophoresis and ScreenTape in this study

\begin{tabular}{|lcc|}
\hline & Manual & ScreenTape \\
\hline No. of PCRs & 20 & 7 \\
Reagent preparation & 45 & 0 \\
Sample loading & 20 & 5 \\
Staining and image capture & 30 & 0 \\
Analysis & 60 & 10 \\
Disposal & 15 & 0 \\
Total and cleaning & 170 & 15 \\
Total time per PCR & 8.5 & 2.1 \\
Total time per isolate $\dagger$ & 127.5 & 31.5 \\
\hline
\end{tabular}

${ }^{\star}$ Includes decontamination to remove ethidium bromide.

$\dagger$ Time required for complete analysis of 15 MIRU-VNTR PCRs.

by the implementation of standardized running conditions, such as temperature control, although this is not a practical option in many settings where TB is a public health problem. Similarly, the discrimination of larger PCR products might be improved by extending their migration through the gel, perhaps by running overnight.

Using current UK list prices, the cost of consumable reagents for sizing one PCR by ScreenTape is approximately 2.40 USD. In our system, running 20 samples per gel, the cost of sizing one PCR by agarose gel electrophoresis is approximately 0.70 USD. A breakdown of hands-on time of ScreenTape, compared to the manual system, is presented in Table 1 . When using ScreenTape, the time required to run and analyse the MIRU-VNTR PCR products was reduced fourfold.

We conclude that the ScreenTape system is a viable alternative to gel electrophoresis for MIRU-VNTR typing of M. tuberculosis isolates. The allele results obtained were comparable to those from a commercial company using a standardized method and an automated sequencer, and allowed rapid differentiation of drug-resistant strains that spoligotyping had initially suggested may have been related. The system could easily be implemented in laboratories which do not have the funds or facilities required to run a highthroughput sequencing machine, thereby enabling MIRU-VNTR typing to be applied more widely in the public health arena of TB control.

\section{Acknowledgements}

The authors wish to acknowledge Imad F. Elmoula, Mohammed S. Ali and Ahammed B. Ali of Al of Neelain University and Nageeb S. Saaed and the Tuberculosis Reference Laboratory, National Health Laboratory, Khartoum, Sudan, for their assistance during the collection of the strains, Lab901 for the loan of the ScreenTape instrument and Donna McDade for training in the use of the instrument. Financial support was received from the Gordon Memorial College Trust Fund and the Department for International Development (UK) funded TARGETS Research Consortium on Communicable Diseases of Vulnerability, Risk and Poverty.

\section{Kim Mallard, ${ }^{1}$ Ghada S. Sharaf Eldin ${ }^{2}$ and Ruth McNerney ${ }^{1}$ \\ ${ }^{1}$ London School of Hygiene and Tropical Medicine, London, UK \\ ${ }^{2}$ Al Neelain University, Khartoum, Sudan}

Correspondence: Kim Mallard

(kim.mallard@Ishtm.ac.uk)

Canetti, G., Froman, S., Grosset, J., Hauduroy, P., Langerova, M., Mahler, H. T., Meissner, G., Mitchison, D. A. \& Sula, L. (1963). Mycobacteria: laboratory methods for testing drug sensitivity and resistance. Bull World Health Organ 29, 565-578.

Dorman, S. E. \& Chaisson, R. E. (2007). From magic bullets back to the magic mountain: the rise of extensively drug-resistant tuberculosis. Nat Med 13, 295-298.

Evans, J. T., Hawkey, P. M., Smith, E. G., Boese, K. A., Warren, R. E. \& Hong, G. (2004)

Automated high-throughput mycobacterial interspersed repetitive unit typing of Mycobacterium tuberculosis strains by a combination of PCR and nondenaturing high-performance liquid chromatography. J Clin Microbiol 42, 4175-4180.

Health Protection Agency Centre for Infections (2008). Tuberculosis in the UK: Annual Report on Tuberculosis Surveillance in the UK 2008. London: Health Protection Agency Centre for Infections.

Kamerbeek, J., Schouls, L., Kolk, A., van Agterveld, M., van Soolingen, D., Kuijper, S., Bunschoten, A., Molhuizen, H., Shaw, R. \& other authors (1997). Simultaneous detection and strain differentiation of Mycobacterium tuberculosis for diagnosis and epidemiology. J Clin Microbiol 35, 907-914.

Kremer, K., Arnold, C., Cataldi, A., Gutierrez, M. C., Haas, W. H., Panaiotov, S., Skuce, R. A., Supply, P., van der Zanden, A. G. \& van Soolingen, D. (2005). Discriminatory power and reproducibility of novel DNA typing methods for Mycobacterium tuberculosis complex strains. J Clin Microbiol 43, 5628-5638.

Mazars, E., Lesjean, S., Banuls, A. L., Gilbert, M., Vincent, V., Gicquel, B., Tibayrenc, M., Locht, C. \& Supply, P. (2001). High-resolution minisatellite-based typing as a portable approach to global analysis of Mycobacterium tuberculosis molecular epidemiology. Proc Natl Acad Sci U S A 98, 1901-1906.

National TB Controllers Association (2004). Guide to the Application of Genotyping to Tuberculosis Prevention and Control. CDC Advisory Group on Tuberculosis Genotyping. Atlanta, GA: US Department of Health and Human Services.

Supply, P., Mazars, E., Lesjean, S., Vincent, V., Gicquel, B. \& Locht, C. (2000). Variable human minisatellite-like regions in the Mycobacterium tuberculosis genome. Mol Microbiol 36, 762-771.

Supply, P., Lesjean, S., Savine, E., Kremer, K., van Soolingen, D. \& Locht, C. (2001).

Automated high-throughput genotyping for study of global epidemiology of Mycobacterium tuberculosis based on mycobacterial interspersed repetitive units. J Clin Microbiol 39, 3563-3571.

Supply, P., Allix, C., Lesjean, S., CardosoOelemann, M., Rusch-Gerdes, S., Willery, E., Savine, E., de Haas, P., van Deutekom, H. \& other authors (2006). Proposal for standardization of optimized mycobacterial interspersed repetitive unit-variable-number tandem repeat typing of Mycobacterium tuberculosis. J Clin Microbiol 44, 4498-4510.

WHO (2008). WHO Report 2008. Global Tuberculosis Control: Surveillance, Planning, Financing. WHO/HTM/TB/2008.393. Geneva: World Health Organization. 